\title{
Obstructive Sleep Apnoea in Orthodontics - A Review
}

\author{
Nandalal Girijalal Toshniwal ${ }^{1}$, Shubhangi Amit Mani², Nilesh Mote ${ }^{3}$, Ashwini Ramesh Nalkar ${ }^{4}$
}

1, 2, 3, 4 Department of Orthodontics, Rural Dental College, Loni, Maharashtra, India.

\section{ABSTRACT}

Obstructive sleep apnoea (OSA) is a sleep associated breathing disorder and it affects the health and quality of life of individuals suffering from it. Orthodontists should be well aware of the symptoms of this disorder and competent enough to recognize its signs and symptoms. Orthodontics is well suited for the treatment of OSA patients due to their expertise and knowledge regarding growth and development of orofacial and dentofacial structures as well as orthopaedic, orthodontic, and surgical correction of the jaws and other supporting tissues.

There are basically two types of sleep apnoea- Central sleep apnoea and obstructive sleep apnoea where obstructive sleep apnoea is the more common one. This disorder can be life threatening as the oxygen supply to various parts of the body is substantially reduced. Obstructive sleep apnoea is caused by an interplay between a variety of factors, including sleep related loss of muscle tone in the tissues supplied by the glossopharyngeal nerve, anatomical obstruction of the nasal passages, large tonsils, large tongue, a retrognathic mandible, obesity, alcohol, sedative medication, and allergies.

Sleep apnoea can be caused due to many factors and many treatment modalities have been employed to correct this disorder including mandibular advancement appliances, polysomnographs, and surgical intervention. It can be treated using surgery, continuous positive airway pressure and oral appliances therapy. This article highlights the role the orthodontist plays in the diagnosis and treatment planning of OSA patients.

\section{KEY WORDS}

Orthodontics, Obstructive Sleep Apnoea, Sleep, Snoring
Corresponding Author: Dr. Ashwini Nalkar, Department of Orthodontics, Rural Dental College, Loni, Maharashtra, India. E-mail: ashu.nalkar@gmail.com

DOI: $10.14260 / \mathrm{jemds} / 2021 / 620$

How to Cite This Article:

Toshniwal NG, Mani SA, Mote N, et al. Obstructive sleep apnoea in orthodontic - $a$ review. J Evolution Med Dent Sci 2021;10(35):3040-3046, $10.14260 /$ jemds/2021/620

Submission 03-03-2021,

Peer Review 14-05-2021,

Acceptance 20-05-2021,

Published 30-08-2021.

Copyright (c) 2021 Nandalal Girijalal Toshniwal et al. This is an open access article distributed under Creative Commons Attribution License [Attribution 4.0 International (CC BY 4.0)] 


\section{BACKGROUND}

Apnoea has origins from Greek meaning "without breath'. Sleep apnoea is known as an intermittent cessation of airflow at the mouth and nose during sleep. Snoring sound is produced by the vibration of the soft palate or other oropharyngeal tissues, and it can become a medical concern because it is a key symptom of OSA. ${ }^{1}$ Duration of apnoea of at least $10 \mathrm{~s}$ has been considered important, but in most patients, they are $15-20 \mathrm{~s}$ in length and may last as long as $1-3$ min..$^{1-3}$ Blood oxygen saturation may decrease abruptly falling below $50 \%$ in severe cases of sleep apnoea. When 30 or more apnoeic episodes occur in the course of seven hours of sleep, resulting in excessive sleepiness during the working hours, a person is described as having sleep apnoea syndrome. This may begin at any age, but incidence increases with age. Anatomic factors that can contribute to OSA are maxillary or mandibular retrognathism, increased lower facial height, large tongue, elongated soft palate and inferiorly positioned hyoid bone. ${ }^{1}$ Neural feedback alerts the body which causes a brief arousal from sleep whenever there is less oxygen.

In 1965 the original description of this condition was written in medical literature. Sleep apnoea can be classified as central, obstructive, and mixed.

\section{TYPES OF APNOEA}

\section{Obstructive Apnoea ${ }^{2}$}

Obstructive sleep apnoea is characterized by the cessation of airflow with persistence of ventilatory effort, caused by collapse of soft tissue structures in the oropharynx or hypopharynx.

\section{Central Apnoea}

Central sleep apnoea (CSA) occurs when the brain temporarily fails to signal the muscles responsible for controlling breathing. Unlike obstructive sleep apnoea, which can be thought of as a mechanical problem, central sleep apnoea is more of a communication problem.

\section{Mixed Apnoea}

Mixed sleep apnoea is a combination of both obstructive and central sleep apnoea symptoms.

In 1918 Sir William Osler coined the term "Pickwickian" to refer to obese, hypersomnolent patients. The name originated from the character of a fat boy named Joe, depicted in the novel by Charles Dickens Posthumous papers of the Pickwick club", who was markedly obese and tended to fall asleep uncontrollably during the day. In 1956 Dr. Burwell and colleagues found similar symptoms of Joe in a patient whom they were treating and termed it as "Pickwickian Syndrome" which is currently called obstructive sleep apnoea. ${ }^{4}$ Central sleep apnoea associated with nerve impulse to all respiratory muscles is absent. Obstructive sleep apnoea (OSA) which is the occlusion of oropharyngeal airway is seen. OSA affects $2-4 \%$ of middle-aged adults. Mixed apnoea is central apnoea followed by obstructive component. Although continuous positive airway pressure (CPAP) is the most efficacious treatment, yet it requires the use of a mask interface, sealed tubing, and a device connected to a power source. This complexity limits its acceptance by patients and leads to suboptimal treatment adherence. Oral appliances are a simpler alternative to CPAP for the treatment of obstructive sleep apnoea (OSA). They are often considered by patients to be a more acceptable treatment modality compared to CPAP, as they are quiet, portable, and do not require a power source. While the role of oral appliances for the treatment of OSA was unclear in the past, this has changed dramatically. ${ }^{1}$

There are various methods of treatment for obstructive sleep apnoea. Pierre Robin who was a French stomatologist, used to treat upper airway obstruction of neonates born with Pierre Robin syndromes with oral appliances. The management of airway obstruction started in 1900 with the use of oral appliances.

\section{Sleep Apnoea Can Be Classified as ${ }^{2}$}

- Central sleep apnoea - Nerve impulse to all respiratory muscles is absent

- Obstructive sleep apnoea (OSA) - Occlusion of oropharyngeal airway is seen. OSA affects $2-4 \%$ of middle-aged adults

- Mixed apnoea - Central apnoea followed by obstructive component.

Obstruction of airflow results in reduction of blood oxygen saturation known as hypoxemia. It produces arousal in an attempt to reopen the airway, due to which the quality of sleep diminishes. This, in turn, results in greatly decreased productivity, time wasting, daydreaming, impaired cognition, increased accident rates, and various other medical disorders that impact every aspect of life.

Consequently, there are partial and complete pauses in breathing that last for at least 10 seconds during sleep. Then, blood oxygen saturation, with oxygen levels decreases abruptly and falls below $50 \%$ or more in severe cases. The brain shows response when there is less oxygen and alerts the body which causes a brief arousal from sleep. This restores normal pattern of breathing. This pattern can occur hundred times in one night. This results in a fragmentation of sleep quality and produces an excessive sleepiness during daytime.

In sleep study monitoring, the frequency of apnoea and hypopneas per hour of sleep [apnoea - hypopnea index (AHI)] is the key measure to define and stratify the severity of OSA, ${ }^{3}$ although inherent limitations to this metric include not taking into consideration the degree of accompanying hypoxia, length of respiratory events, etc. AHI levels of 5, 15 and 30 have been used as cut-points to define mild, moderate, and severe OSA, respectively. Apnoea can be distinguished as obstructive vs. central based upon the presence or absence of thoracoabdominal effort.

\section{Normal Sleep}

Normal sleep architecture is characterized by two forms, namely non-rapid eye movement (NREM) and rapid eye movement (REM). ${ }^{2}$ Sleep is initiated in stage 1 NREM and progressively moves through the deeper stages 2,3 , and 4 
before reaching REM sleep. REM sleep is associated with vivid dreaming and diminished tone of the skeletal muscles of the pharyngeal space and limbs.

During NREM sleep, breathing frequency and aspiratory flow rate are reduced with minute ventilation falls. In the deepest stage of NREM sleep (stage 4), breathing is slow, deep, and very regular. However, in stages 1 and 2, the depth of breathing sometimes varies periodically. The explanation is that in light sleep, removal of the wakefulness stimulus varies over time in a periodic fashion: When removed, sleep is deeper and breathing is depressed. When returned to normal, breathing is excited not only by the wakefulness stimulus but also by the carbon dioxide gas which was retained during the interval of sleep. This pattern of breathing is known as Cheyne-Stokes respiration.

\section{Prevalence}

OSA occurs mostly in children with prevalence being nearly 2 $\%$. Prevalence of OSA increases with age, in at least $4 \%$ and 2 $\%$ in men and women respectively. OSA leads to excessive daytime sleepiness. Breathing symptoms of OSA with or without daytime sleepiness occur in $24 \%$ of men and $9 \%$ of women.

\section{Etiological Factor of $\mathrm{OSA}^{5}$}

- Obesity.

- Retropositioned maxilla and mandible.

- Mandibular deficiency.

- Macroglossia.

- Adenotonsillar hypertrophy.

- Upper airway tumours.

- Loss of muscle tone.

- Nasal Obstruction.

- Alcohol.

- Sedative medications (eg. benzodiazepines - valium, ativan).

- $\quad$ Narcotics (codeine, morphine).

\section{Symptoms}

- Loud snoring.

- Apnoeic pauses that manifest as gasping, snoring, and choking during the night

- Behavioural disturbances.

- Restless sleep.

- Increase in body movements.

- Bruxism

- Disturbed sleep.

- Cerebral hypoxia at night.

- Narcolepsy like symptoms.

- Impaired intellectual performance.

- Poor concentration, and distraction.

- Memory loss.

- Impotence in men.

- Poor school performance.

- Infraorbital venous congestion.

\section{PATHOPHYSIOLOGY OF ORAL SLEEP APNOEA}

It has been hypothesized genioglossal that individuals with OSA have impaired genioglossal function, allowing the prolapse of the tongue against the posterior pharyngeal wall with inspiratory effort during sleep. The situation now appears to be more complicated - evidence suggests that an invagination of the pharyngeal walls and a general hypotonia of the dilating muscles of the upper airway can also be involved in allowing airway occlusion during sleep. The nasal airway can also play an important role in total airway occlusion. Nasal obstruction increases resistance to air flow, which in turn results in increased inspiratory effort and greater negative pressure in the pharyngeal airway. ${ }^{1}$

The resulting apnoea leads to progressive asphyxia until there is a brief arousal from sleep, whereupon airway patency is restored and airflow resumes. The patient then returns to normal sleep, and this cycle of events is repeated. The immediate factor leading to collapse of the upper airway in OSA is the production of critical sub-atmospheric pressure during inspiration that exceeds the potential of the airway dilator and abductor muscles to maintain airway stability. Obesity frequently contributes to the reduction in the size of upper airway, either by increasing fat deposition in the soft tissues around the pharyngeal airway or by compressing the pharynx by superficial fat masses in the neck. Snoring, a highfrequency vibration of the palatal and pharyngeal soft tissues that results from the decrease in size of the upper airway lumen, may aggravate the narrowing by producing oedema of the soft tissues. ${ }^{2}$

\section{Diagnosis}

The diagnosis is made by the following ways:

- Physical and oral examination -

- Sleep history

- Epworth sleepiness scale questionnaire

- Apnoea hypopnea index (AHI)

- $\quad$ Polysomnography (PSG).

\section{Physical and Oral Examination}

Methods of Assessing the Degree of Respiratory Obstruction ${ }^{2}$ The methods are thorough history and physical examination with regard to sleeping habits, snoring, mouth breathing, hypersomnia, headaches, lethargy, weight gain and difficulty in awakening. Physical examination of the head, neck, chest, and abdominal areas include the examination of respiratory, cardiovascular, nervous system and upper pharynx. Assessment of the BMI (risk factor for OSA is above $30 \mathrm{~kg} /$ $\mathrm{m} 2$ ) \& the circumference of neck (above 17 inches in men, or 16 inches in women is a risk factor for OSA) is of utmost importance. Intraorally the patient should also be examined for micrognathia, tonsillar hypertrophy, and lateral peritonsillar narrowing, macroglossia, elongated or enlarged uvula, nasal septal deviation, and nasal polyps, high arched or narrow palate. For determining the difficulty of performing an intubation as the tongue obstructs the airway, Malampati scores are used in anaesthesia. 6 (mallampatti scores of $3 \& 4$ exhibit greater risk of sleep apnoea), Possible sites of obstruction (Mallampati et al. 1985) ${ }^{4}$ 
Nose

- Deviated septum

- $\quad$ Enlarged turbinates

- $\quad$ Polyps

- Nasopharynx

- $\quad$ Enlarged adenoids

- Pharynx

- Enlarged tonsils

- Enlarged uvula or soft palate

- Enlarged base of the tongue

- Tongue base falling into pharyngeal airway

- Submucosal fat or redundant mucosa

The upper airway should be examined in all patients, particularly in non-obese adults who exhibit symptoms consistent with those of OSA.

\section{Polysomnography}

It is considered the gold standard of sleep apnoea diagnosis (Farney et al. 2011). A sleep technician administered the study. Sleep polysomnography features electrocardiography / EKG, brainwave electroencephalography / EEG measurements, motor activity extremity measurements, diaphragmatic / chest movement, eye movement, pulse oximetry for oxygen desaturation measurement, and inhalational / exhalational oronasal flow characteristics. It plays a vital role in the diagnosis of sleep apnoea. PSG involves an overnight observation and monitoring of multiple channels and physiologic variables in the presence of a technician. For the duration of the study, sleep stages, sleep continuity, respiratory effort, oxygen saturation, electrocardiogram, airflow, body position, and movements are recorded.8-9

\section{Criteria Included}

- Cessation of air flow for 10 seconds even with maintenance of respiratory effort.

- Five or more episodes of apnoea per hour.

- Decrease in oxygen saturation of at least $4 \%$ during episodes.

Cephalometric characteristics of OSA (Alan lowe)

- Mandibular retrognathia

- Retruded maxilla

- Posterior vertical maxillary deficiency

- Retropositioned tongue

- High mandibular plane angulation

- Short chin - neck line

- Poor definition of gonial angles

- Class II dental occlusion (but sometimes Class I)

- Longer soft palate

\section{oximetry}

Overnight oximetry helps in assessing abnormal drops in oxygen level which occur recurrently in sleep apnoea. OSA is often associated with periodic pauses in breathing and drops in the oxygen level of the blood. Overnight oximetry is an easy and inexpensive method of screening test which helps in assessing abnormal drops in oxygen level.

\section{Multiple Sleep Latency Test (MSLT)}

It is an examination to test how quickly a person falls asleep during the daytime. It consists of 4 - 5 naps of 20 minutes' duration, at every 2 hours interval during the day. On an average an adult requires a mean sleep latency period of 10 minutes or more. Individuals who exhibit excessive day time sleepiness show a mean latency period of less than 5 minutes.

\section{EMG Activity}

Patients suffering from OSA when moved to a supine position from an upright posture causes the tongue to fall backwards and results in the blockade of the airway. When the patient is asleep, the movement of the tongue is reduced, and this reduction of the tone leads to the thickening of the muscle and may also cause backward positioning of the tongue. Pharyngeal wall (Kuna et al. 1997) - A study done by Kuna et al. evaluated the pharyngeal constrictor muscles of normal adults during their wakefulness and sleep. The electromyographic activity of these muscles were checked upon and was concluded that there was a decreased electromyographic activity of these muscles which indicates a decreased tone of the same.

\section{Apnoea Hypopnea Index (AHI)}

The severity of OSA is assessed by the frequency of pauses in breathing on an hourly basis and it is known as AHI or as the respiratory disturbance index. ${ }^{10}$

\begin{tabular}{|ccc|}
\hline SL. No. & $\begin{array}{c}\text { Types of Subjective } \\
\text { Daytime Sleepiness }\end{array}$ & ESS Number \\
1 & Normal & ESS $<11$ \\
2 & Mild & ESS $=11$ \\
3 & Moderate & ESS $=16$ \\
4 & Severe & ESS $>18$ \\
\hline \multicolumn{2}{|c|}{ Table 1. Apnoea Hypopnea Index Score for Adults } \\
\hline
\end{tabular}

\section{Treatment}

The treatment options for OSA could be broadly classified into Non-specific and specific therapy. ${ }^{4}$

\section{Nonspecific Therapy}

As the name suggests, this treatment should be advised for all the patients suffering from OSA no matter what the severity is but it is most notably used for patients whose chief concern is snoring with a mild apnoea.

\section{Specific Therapy}

Specific therapy on the other hand is tailored to the individual patients need based on medical history, physical examination, and the results obtained from polysomnography. There are various factors to be assessed before selecting the treatment modalities for a specific individual. These include -

- The severity of the disease

- The obstruction site

- Subjective symptoms

- Patient preference.

\section{Behavioural Interventions}

Weight reduction therapy can be advised to patients with obstructive sleep apnoea who are obese, therefore it improves 
symptoms of OSA. Smokers should be advised to stop smoking. Patients suffering from mild OSA can find relief with positional therapy. Reduced symptoms can be achieved with raising the head of the bed or by sleeping in a supine manner. ${ }^{12}$

\section{NON-SURGICAL OPTIONS}

\section{Oxygen Administration}

Not commonly used alone but when used in combination with nasal continuous positive airway pressure it corrects this problem. It's usually used in central apnoea which is caused by heart failure rather than in patients with obstructive sleep apnoea.

\section{Positive Pressure Therapy}

This is one of the most effective treatment therapies for OSA. It includes:

- $\quad$ Continuous Positive Airway Pressure (CPAP)

- Autotitration

- Bi - level positive airway pressure

Continuous Positive Airway Pressure (CPAP) CPAP is a mask-like device that fits snugly over the patient's nose. To hold the throat open, for the duration of sleep a continuous stream of positively pressurized air is sent that is customized for each person. It helps in keeping the airway patent during sleep breathing. CPAP may rarely cause paranasal sinusitis and significant epistaxis.

\section{Autotitration}

These are the devices which are designed such that they provide the minimally required pressure at a certain period and also change the pressure as the patients' requirements change.

\section{Bi - Level Positive Airway Pressure}

This is similar to CPAP but has an advantage over it as it is designed in such way that it automatically detects the patients breathing pattern and modifies the pressure on inhalation and exhalation.

\section{Pharmacological Agents}

Various pharmacological agents may also be used but are said to be not very useful for the treatment of sleep apnoea. These include:

- Thyroid hormone supplementation

- Progestational agents like oestrogen

- acetazolamide

- Tricyclic antidepressants

- modafinil.

\section{Oral Appliance Therapy}

Oral appliances can alleviate the symptoms of OSA in 2 ways. They can enlarge the 3 dimensional volume of the upper airway or by reducing the collapsibility of the soft tissues in the region ensuring patency during sleep. Oral appliances include mandibular advancement appliances and tongue retaining devices which are splints that hold the tongue in place to keep the airway patent at all times.15-16 These appliances allow continuous breathing during sleep by bringing the tongue and mandible forward by opening up of the upper airway.

\section{Mandibular Advancement Appliances}

The ideal properties of removable appliances include simplicity of delivery, low bulk, lip seal maintenance, sufficient tongue space, non-interference with sleep, low cost, and lateral freedom. The patients selected for these types of appliances need to have certain features for the appliance to exhibit the best possible results. These include reduced lower anterior facial proportions, normal relation between maxilla and mandible, high position of hyoid, normal soft palate area and tongue proportion, and relatively normal postpalatal and postlingual airway. Advancement appliances are manufactured in different materials and sold under different trade names. Their main purpose is to place the mandible in a forward position so that the size of the airway passage is increased. Kyung et al. measured the pharyngeal size and shape differences between pre and post trials of a mandibleprotruding oral appliance using cine computerized tomography. They found that the oral appliances appeared to enlarge the pharynx to a greater degree in the lateral than in the sagittal plane at the retropalatal and retroglossal levels of the pharynx, thus suggesting a mechanism for the effectiveness of oral appliances that protrude the mandible. Ngiam and Kyung investigated the efficacy of orthodontic microimplant - based mandibular advancement treatments for the management of severe snoring and OSA in adult patients. They found that favourable reduction in sleep variables highlighted the potential of microimplant - based mandibular advancement therapy as an alternative treatment modality for OSA patients who cannot handle the continuous positive airway pressure and oral appliance therapy. Referring to these studies, it is clear that the advancement appliances have given very favourable results in the treatment of OSA.

Tongue retaining devices were first developed in 1979. They are made up of soft polyvinyl. This bubble shaped appliance has custom fitted grooves in which the patient's teeth rest while the tongue rests within the bubble. The bubble forms a suction that holds onto the tongue in a more anterior position and hence any obstruction due to the base of the tongue is avoided. ${ }^{4}$

\section{Oral Airway Dilator Designs}

1. The Silencer System: This appliance incorporates the Halstrom Hinge Titanium Precision Attachment at the incisor level, it permits $2 \mathrm{~mm}$ advancement up to $8 \mathrm{~mm}$, a lateral movement of $6 \mathrm{~mm}, 3 \mathrm{~mm}$ bilaterally, and vertical pin height replacements. A flat posterior bite plane is provided for the biting surfaces. This appliance cannot be adjusted by the patient, but must be adjusted in the dental office. It is made of elastomeric plastic and is the only appliance that allows adjustment in not only a front to back position, but also in an 'open and close' position. It is one of the most expensive appliances due to the presence of the titanium hinge.

2. The PM Positioner: It contains bilateral orthodontic expanders which link upper and lower, and have 
attachment connectors on both lateral sides (Lílian et al. 2008). This appliance is made of a thermoplastic material which must be heated in hot tap water every night before it is placed in the mouth. This appliance is bound firmly on the buccal side of the molar teeth and restricts the mandible once worn.

3. Mandible repositioning device: It likely has a role as a first - line therapy for the treatment of mild and moderate OSA patients and as a second - line therapy for severe patients (Stuart et al. 1996)

4. The Klearway oral appliance: This appliance is fabricated using a thermoactive acrylic, it becomes pliable for easy insertion and confirms securely to the dentition for an excellent fit while significantly decreasing soft tissue and tooth discomfort. It utilizes a maxillary expander to sequential advancement of the mandible. Klearway is a fully adjustable oral appliance used for the correction of mild to moderate obstructive sleep apnoea and snoring. Some amount of jaw movements, i.e., the vertical and lateral direction is permitted without dislodging the appliance.

5. The Elastic Mandibular Advancement (EMA): Compared to the other appliances the EMA appliance is the least bulky and gives about significant amount of freedom for the jaw movements (Henke et al., 2000). It is not very patient friendly and may be difficult to tolerate.

\section{Indications}

1. Patients with snoring or mild OSA who do not respond for treatment with behavioural measures.

2. Patients with moderate to severe OSA who refuse treatment with nasal CPAP.

3. Patients who are not appropriate for tonsillectomy, adenoidectomy, and tracheostomy.

\section{Mechanism of Action}

Oral appliances are used only during sleep which repositions the lower jaw, tongue, soft palate or uvula and maintains an open and unobstructed airway. It protrudes the mandible and tongue forwards and prevents upper airway collapse during the sleep.

\section{Advantages of Oral Appliances}

1. Patients for whom surgical intervention is contraindicated.

2. Patients refusing CPAP who have moderate to severe OSA.

3. Patients with snoring or mild OSA who are noncooperative to behavioural measures.

\section{Disadvantages of Oral Appliances}

Forces generated of teeth and jaw by the mandibular splint resulting in salivation, headaches, gum soreness, temporomandibular pain, dry mouth and tooth pain. 17 - 18

\section{SURGICAL OPTIONS}

Various surgical approaches are in the contemporary vogue in the treatment of OSA.

\section{Surgical Measures}

- Uvulopalatopharyngoplasty (UPPP): It is the reconstruction of the throat by resection of posterior margins of the soft palate and unwanted mucosa present on the pharyngeal walls

- Adenotonsillectomy: It is the surgical removal of the tonsils and adenoids and is the most common treatment option for children with OSA.

- Tracheostomy: It was the first surgical treatment for OSAHS and bypasses the obstruction completely

- Midline glossectomy

- Maxillomandibular osteotomy and advancement.19

- $\quad$ Other surgical techniques a) Bariatric (weight reducing) surgery: Weight influences the severity of OSAHS and weight loss is an effective treatment for OSAHS in some patients. b) Nasal surgery: Nasal surgery decreases nasal airflow resistance and reduces pressure and improves compliance with nasal CPAP. ${ }^{3}$

\section{CONSEQUENCES OF UNTREATED} ORAL SLEEP APNOEA

Obstructive sleep apnoea negatively impacts quality of life and is also associated with a number of adverse safety and health consequences including cardiovascular disease and motor vehicle crashes. Short habitual sleep duration can result in excessive daytime sleepiness and reduced neurocognitive function. Sleep loss may have long term health consequences and may lead to premature death, cardiovascular disease, and the development of diabetes. Patients may also experience impaired concentration due to tiredness, increased irritability, depression and mood changes. There is an increased risk of high blood pressure and may have a slightly increased risk of angina, heart attacks and strokes. ${ }^{3}$

\section{CONCLUSIONS}

As orthodontists we play a significant role in the diagnosis and evaluation of sleep apnoea. Various factors should be kept in mind while formulating the treatment plan. Sleep apnoea affects daily activities of the individual affecting the cognitive function, personality changes, increased mood swings and narcolepsy - like symptoms.

Financial or other competing interests: None.

Disclosure forms provided by the authors are available with the full text of this article at jemds.com. 


\section{REFERENCES}

[1] Kumar NS, Divya K, Naragond A, et al. Obstructive sleep apnoea-an orthodontic review. IOSR Journal of Dental and Medical Sciences (IOSR-JDMS) 2013;9(6):68-72.

[2] Kulshrestha R, Rohmetra A. Obstructive sleep apnoea in orthodontics: an overview. International Journal of Orthodontic Rehabilitation 2016;7(3):115-8.
[3] Agarwal L, Gupta A. Role of orthodontist in obstructive sleep apnoea-an orthodontic review. Journal of Orthodontics \& Endodontics 2016;2(3)10:1-7.

[4] George M, Nadeem F, Samson S, et al. Obstructive sleep apnoea and orthodontics-a review. International Journal of Current Research 2019;11(4):3117-20. 